\title{
Reentrant phase transition of Born-Infeld-dilaton black holes
}

\author{
Seyed Hossein Hendi ${ }^{1,2, a}{ }_{\mathbb{C}}$, Mehrab Momennia ${ }^{1, b}$ \\ ${ }^{1}$ Physics Department and Biruni Observatory, College of Sciences, Shiraz University, Shiraz 71454, Iran \\ ${ }^{2}$ Research Institute for Astronomy and Astrophysics of Maragha (RIAAM), P.O. Box 55134-441, Maragha, Iran
}

Received: 20 June 2018 / Accepted: 21 September 2018 / Published online: 1 October 2018

(C) The Author(s) 2018

\begin{abstract}
We explore a novel reentrant phase transition of four-dimensional Born-Infeld-dilaton black hole in which the first order phase transition modify into a zeroth order phase transition below the critical point. Working in the extended phase space with regarding the cosmological constant as a pressure, we study the reentrant behavior of phase transition in the canonical ensemble. We show that these black holes enjoy a zeroth order intermediate-small black hole phase transition as well as a first order phase transition between small and large black holes for a narrow range of temperatures and pressures. We also find that the standard first order small-large black hole phase transition can modify into a zeroth order type. This zeroth order phase transition stands between the critical point and the first order phase transition region. We discuss the significant effect of the scalar field (dilaton) on the mentioned interesting treatment.
\end{abstract}

\section{Introduction}

The phase transition is one of the interesting topics in thermodynamical systems. During the phase transition, we often encounter with discontinuity of a certain thermodynamic property. In order to classify the phase transition systematically, we can concentrate on the Gibbs thermodynamic potential and its first and higher derivatives. In the paradigm of the phase transition, one may use some analogies and symmetries between usual thermodynamic systems and black holes to deepen his insight into the black hole physics. It was shown that black holes can be considered as thermodynamical systems [1-3] with typical temperature [4] and entropy [5] which satisfy the first law of black hole thermodynamics [6]. It has also been found that they can behave like ordinary thermodynamical systems and undergo the phase transition [7]. A more interesting case was observed when one con-

\footnotetext{
a e-mail: hendi@shirazu.ac.ir

be-mail: m.momennia@shirazu.ac.ir
}

siders a correspondence of variables $(Q, \Phi) \leftrightarrow(P, V)$ for charged black holes which results into the van der Waals like phase transition [8-10]. In recent years, the idea of considering the cosmological constant as thermodynamical pressure has attracted a lot of attention in black hole thermodynamics $[11,12]$

$P=-\frac{\Lambda}{8 \pi}$,

where the thermodynamical quantity conjugate to $P$ is the thermodynamical volume

$V=\left(\frac{\partial M}{\partial P}\right)_{r e p}$,

in which "rep" stands for "residual extensive parameters". The motivation comes from the fact that in some fundamental theories there are several physical constants, such as Yukawa coupling, gauge coupling constants, and Newton's constant that are not fixed values. On the other hand, the cosmological constant stands by pressure side in Tolman-OppenheimerVolkoff equation which shows the cosmological constant can be considered as thermodynamical pressure. Considering $\Lambda$ as the pressure of system leads to a van der Waals like smalllarge black holes (SBH-LBH) phase transition which has been investigated by so many authors (for instance see incomplete list [13-23] and references therein).

The reentrant phase transition (RPT) can occur in a thermodynamical system whenever a monotonic change of any thermodynamic variable leads into more than one phase transition so that the final state is macroscopically similar to the initial state. In our black holes case study, there is a specific range of temperatures such that black holes undergo a large-small-large phase transition by a monotonic changing of the pressure. This interesting phenomenon has been first observed in a nicotine-water mixture [24], and then seen in multicomponent fluids, binary gases, liquid crystals, and other diverse systems [25]. In the context of black holes, 
the RPT has been observed for Born-Infeld adS black holes [26], rotating adS black holes [27,28], dS black holes [29], hairy black holes [30], and adS black holes in massive gravity $[31,32]$. The van der Waals like phase transition of SBHLBH in dilaton gravity has been investigated for charged adS black holes [33], and also, different types of nonlinear electrodynamics, such as power Maxwell invariant [34,35], exponential [36], and Born-Infeld [37,38]. More recently, a study regarding zeroth order SBH-LBH phase transition of charged dilaton black holes has been done [39]. The purpose of this paper is studying the thermodynamics of 4dimensional Born-Infeld-dilaton black holes and investigating the RPT in the canonical ensemble of extended phase space. In addition, we are going to show that this kind of black holes can undergo a zeroth order SBH-LBH phase transition.

\section{Review of solutions and thermodynamics}

Topological Born-Infeld-dilaton black holes in 4-dimensional spacetime were constructed by Sheykhi [40]. In what follows we concentrate our attention on the spherical symmetric black holes with negative cosmological constant. The line element reads

$$
\begin{aligned}
d s^{2}= & -f(r) d t^{2}+f^{-1}(r) d r^{2} \\
& +r^{2} R^{2}(r)\left(d \theta^{2}+\sin ^{2} \theta d \varphi^{2}\right),
\end{aligned}
$$

where $f(r)$ and $R(r)$ are given by

$$
\begin{aligned}
f(r)= & -\frac{\alpha^{2}+1}{\alpha^{2}-1}\left(\frac{b}{r}\right)^{-2 \gamma}-\frac{m}{r^{1-2 \gamma}} \\
& +\frac{\left(\alpha^{2}+1\right)^{2} r^{2}}{\alpha^{2}-3}\left(\frac{b}{r}\right)^{2 \gamma}\left\{\Lambda+2 \beta^{2}\right. \\
& \left.\times\left[{ }_{2} \mathcal{F}_{1}\left(-\frac{1}{2}, \frac{\alpha^{2}-3}{4}, \frac{\alpha^{2}+1}{4},-\eta\right)-1\right]\right\}, \\
R(r)= & e^{\alpha \Phi},
\end{aligned}
$$

in which $\alpha$ is an arbitrary dilaton coupling constant determining the strength of coupling of the scalar and electromagnetic field, $b$ is an arbitrary constant, $\beta$ is nonlinearity (Born-Infeld) parameter, and ${ }_{2} \mathcal{F}_{1}$ is a hypergeometric function. In addition, $\gamma=\frac{\alpha^{2}}{\alpha^{2}+1}, \eta=\frac{q^{2}}{\beta^{2} r^{4}}\left(\frac{r}{b}\right)^{4 \gamma}$, and $\Phi$ is the dilaton field

$\Phi(r)=\frac{\gamma}{\alpha} \ln \left(\frac{b}{r}\right)$.

Using the definition of the surface gravity, one can obtain the Hawking temperature of the black hole on the outermost horizon, $r_{+}$,

$$
\begin{aligned}
T= & \frac{\alpha^{2}+1}{2 \pi r_{+}\left(\alpha^{2}-1\right)}\left(\frac{b}{r_{+}}\right)^{2 \gamma}\left[r_{+}^{2}\left(\alpha^{2}-1\right)\left(\beta^{2}-\frac{\Lambda}{2}\right)\right. \\
& \left.-\frac{1}{2}\left(\frac{r_{+}}{b}\right)^{4 \gamma}-\beta^{2} r_{+}^{2}\left(\alpha^{2}-1\right) \sqrt{1+\eta_{+}}\right],
\end{aligned}
$$

where $\eta_{+}=\left.\eta\right|_{r=r_{+}}$. In addition, the entropy of the black hole per unit volume $V_{2}$ in Einstein gravity is a quarter of the event horizon area

$S=\frac{A}{4}=\frac{r_{+}^{2}}{4}\left(\frac{b}{r_{+}}\right)^{2 \gamma}$.

The electric potential $U$, measured at infinity with respect to the horizon is given by the following explicit form

$U=\frac{q}{r}{ }_{2} \mathcal{F}_{1}\left(\frac{1}{2}, \frac{\alpha^{2}+1}{4}, \frac{\alpha^{2}+5}{4},-\eta_{+}\right)$,

where $q$ is an integration constant which is related to the electric charge of the black hole. One can use the flux of the electric field at infinity to obtain the electric charge per unit volume $V_{2}$

$Q=\frac{q}{4 \pi}$.

The total mass of obtained black holes per unit volume $V_{2}$ can be obtained by using the behavior of the metric at large $r$

$M=\frac{m b^{2 \gamma}}{8 \pi\left(\alpha^{2}+1\right)}$.

It has been shown that by considering the entropy and electric charge as a complete set of extensive parameters, these conserved and thermodynamical quantities satisfy the first law of thermodynamics [40]

$d M=T d S+U d Q$.

\section{Reentrant phase transition}

In the extended phase space, the negative cosmological constant is considered as a positive thermodynamical pressure $[11,12]$, which in our case it has the following form

$P=-\frac{\Lambda}{8 \pi}\left(\frac{b}{r_{+}}\right)^{2 \gamma}$.

It is worthwhile to mention that in order to find the relation between the cosmological constant and the pressure, one can use the components of energy-momentum tensor in diagonal form. Regarding the energy-momentum tensor, we find that 
although in the absence of dilaton field the traditional definition of pressure $(P=-\Lambda /(8 \pi))$ is obtained, such definition may be generalized in the presence of dilaton field. It is worth mentioning that if one uses the traditional relation of the pressure in the presence of dilaton gravity, the same van der Waals like phase transition may be seen, but with different critical quantities, which is not correct.

As we mentioned before, in the absence of dilaton field $(\gamma=0)$, the pressure (13) takes the standard form (1). In this situation, the total mass (11) plays the role of enthalpy of system [12], and the Smarr formula is given by

$$
\begin{aligned}
M & =2(1-\gamma) T S+U Q+(2 \gamma-1)(2 V P+\mathcal{B} \beta) ; \\
\mathcal{B} & =\left(\frac{\partial M}{\partial \beta}\right)_{S, Q, P} .
\end{aligned}
$$

The validity of the first law of thermodynamics in the extended phase space should be written as a differential equation for the enthalpy with the following form

$d M=T d S+U d Q+V d P+\mathcal{B} d \beta$,

where $V$ is the thermodynamical volume conjugate to $P$

$$
V=\left(\frac{\partial M}{\partial P}\right)_{S, Q, \beta}=\frac{\alpha^{2}+1}{3-\alpha^{2}} b^{2 \gamma} r_{+}^{\left(\alpha^{2}+3\right) /\left(\alpha^{2}+1\right)} .
$$

On the other hand, although it is possible to add some additional terms like $B d b(B=\partial M / \partial b)$ and $A d \alpha(A=\partial M / \partial \alpha)$ to the (differential form of) first law, mathematically, we are not allowed to do this. It is expected that all intensive and extensive thermodynamic parameters appear in the Smarr formula (14). Therefore, we considered $b$ and $\alpha$ as two constants (not thermodynamic variables) since they do not appeared in the Smarr formula.

Here, we study the thermodynamics of 4-dimensional Born-Infeld-dilaton black holes in the canonical ensemble (fixed $Q$ and $\beta$ ) of extended phase space. So, by using the temperature (7) and the relation between the cosmological constant and pressure (13), it is straightforward to show that the equation of state, $P=P\left(r_{+}, T\right)$, is given by

$$
\begin{aligned}
P= & \frac{T}{2 r_{+}\left(\alpha^{2}+1\right)}+\frac{1}{8 \pi r_{+}^{2}}\left[\frac{1}{\left(\alpha^{2}-1\right)}\left(\frac{b}{r_{+}}\right)^{-2 \gamma}\right. \\
& \left.-2 \beta^{2} r_{+}^{2}\left(\frac{b}{r_{+}}\right)^{2 \gamma}\left(1-\sqrt{1+\eta_{+}}\right)\right] .
\end{aligned}
$$

The thermodynamical behavior of the system is governed by the Gibbs free energy, therefore, we should obtain the Gibbs free energy as well. In the extended phase space, one can determine the Gibbs free energy per unit volume $V_{2}$ by using the following definition

$$
\begin{aligned}
G= & M-T S \\
= & \frac{1}{16 \pi}\left\{2 \beta^{2} r_{+}^{3}\left(\alpha^{2}+1\right)\left(\frac{b}{r_{+}}\right)^{4 \gamma}\left(\sqrt{1+\eta_{+}}-1\right)\right. \\
& -\frac{4\left(\alpha^{2}+1\right)}{\left(\alpha^{2}-3\right)} b^{4 \gamma} r_{+}^{\left(3-\alpha^{2}\right) /\left(\alpha^{2}+1\right)} \\
& \times\left[4 \pi P\left(\frac{b}{r_{+}}\right)^{-2 \gamma}+\beta^{2}(1-\mathcal{H})\right] \\
& \left.-r_{+}\left[8 \pi r_{+}^{2} P\left(\alpha^{2}+1\right)\left(\frac{b}{r_{+}}\right)^{2 \gamma}-1\right]\right\},
\end{aligned}
$$

where $\mathcal{H}={ }_{2} \mathcal{F}_{1}\left(-\frac{1}{2}, \frac{\alpha^{2}-3}{4}, \frac{\alpha^{2}+1}{4},-\eta_{+}\right)$.

On the other hand, the heat capacity in extended phase space at constant pressure is

$C_{P}=T\left(\frac{\partial S}{\partial T}\right)_{P}$,

which can be obtained easily by using Eqs. (7) and (8). It is worthwhile to mention that the heat capacity at constant $P$ is, in fact, a heat capacity at constant $P, Q$, and $\beta$ because we are working in the canonical ensemble. The negativity of $C_{P}$ represents unstable black holes, whereas its positivity indicates stable ones. In order to study the phase transition of black holes, one can use the definition of inflection point

$$
\left.\frac{\partial P\left(r_{+}, T\right)}{\partial r_{+}}\right|_{T=T_{c}, r_{+}=r_{+c}}=\left.\frac{\partial^{2} P\left(r_{+}, T\right)}{\partial r_{+}^{2}}\right|_{T=T_{c}, r_{+}=r_{+c}}=0,
$$

which can be used to obtain the critical horizon radius $r_{+c}$ and temperature $T_{c}$. Due to the complexity of obtained relation, we cannot calculate $r_{+c}$ analytically. So, we use the numerical method and some diagrams to investigate the RPT of the black holes for the fixed values of $q=0.2, \alpha=0.1$, $\beta=2$, and $b=1$.

The general behavior of Born-Infeld-dilaton black holes is illustrated in Figs. 1, 2, and 3. Figure 1 (2) has been plotted for different regions of temperature (pressure) in $P-r_{+}$ $(G-T)$ diagram, and Fig. 3 shows the coexistence lines. The different areas of temperature and pressure in Figs. 1 and 2 are equivalent to each other. For $P>P_{c}$ in $G-T$ diagram, the curve looks like the Hawking-Page phase transition [7]. The dashed red line describes small unstable black holes with the negative heat capacity whereas the solid line corresponds to stable large black holes with the positive heat capacity (for more discussion about the relation between the heat capacity and Gibbs energy see [23]). Considering Figs. 1 and 2, one can see that there is a critical point at $P=P_{c}$ in $G-$ $T$ diagram (at $T=T_{c}$ in $P-r_{+}$diagram which characterized by an inflection point) which shows a second order 


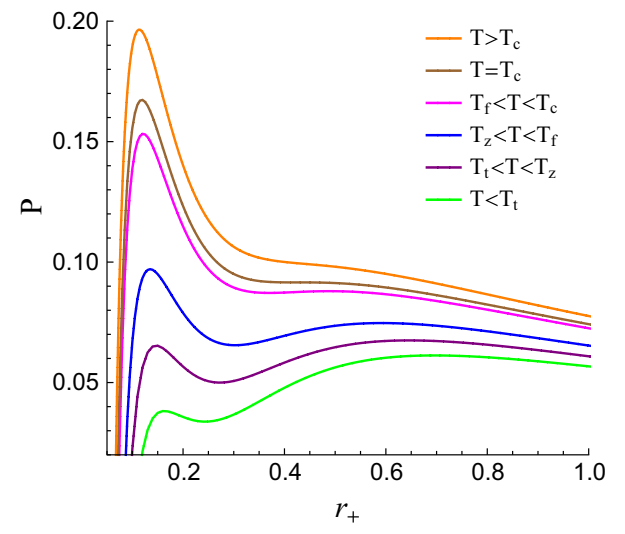

Fig. $1 P-r_{+}$diagram for different regions of temperature. At $T=T_{c}$ there is an inflection point which shows a second order phase transition between $\mathrm{SBH}$ and $\mathrm{LBH}$, and black holes are physically indistinguishable for $T>T_{c}$. For $T_{f}<T<T_{c}\left(T_{z}<T<T_{f}\right)$, black holes undergo a zeroth (first) order SBH-LBH phase transition. The RPT is located at $T_{t}<T<T_{z}$ and black holes undergo the LBH-SBH-IBH phase transition in this region of temperature. There are $\mathrm{LBH}$ at $T<T_{t}$ and there is no phase transition in this region

phase transition between SBH and LBH. For $P_{f}<P<P_{c}$ $\left(T_{f}<T<T_{c}\right)$, there is a region in which black holes undergo a zeroth order SBH-LBH phase transition. The vertical line at $T=T_{0} \in\left(T_{f}, T_{c}\right)$ in Fig. 2 shows this behavior with a finite jump in the Gibbs energy. This kind of phase transition has never seen before in the RPT of black holes, and it is due to the presence of the dilaton field (for more details see Table 1 and related discussion). The standard first order SBH-LBH phase transition occurs for $P_{z}<P<P_{f}$ $\left(T_{z}<T<T_{f}\right)$. For $P \in\left(P_{t}, P_{z}\right)$ and $T \in\left(T_{t}, T_{z}\right)$, there are three different phases of intermediate black holes (IBH), $\mathrm{SBH}$, and $\mathrm{LBH}$. The vertical line at $T=T_{1} \in\left(T_{t}, T_{z}\right)$ in Fig. 2 shows a discontinuity in the Gibbs free energy which describes a zeroth order phase transition between SBH and

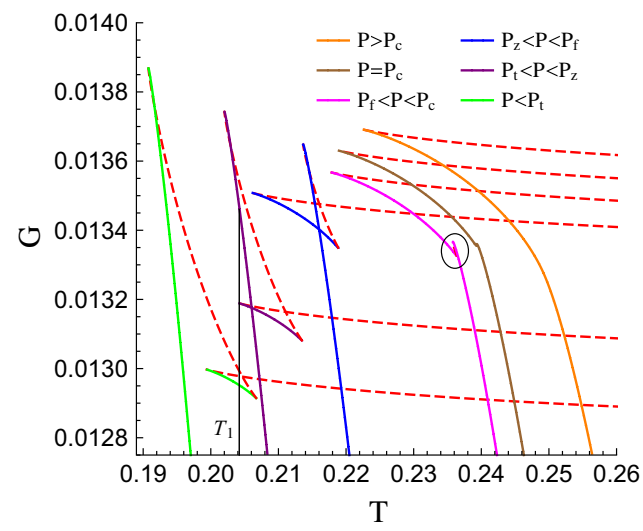

Fig. $2 G-T$ diagram for different regions of pressure. The right panel indicates a close-up of the zeroth order SBH-LBH phase transition located at the small circle in the left panel. The solid lines correspond to $C_{P}>0$ whereas the dashed red lines are related to $C_{P}<0$. At the
IBH. There is also a first order SBH-LBH phase transition in this region of pressures and temperatures. This behavior is known as RPT. It is worthwhile to mention that IBH are macroscopically similar to LBH. Therefore, black holes undergo the large-small-large phase transition in this region of pressures. Finally, there are just LBH for $P<P_{t}$ and $T<T_{t}$.

Figure 3 shows the coexistence lines of SBH+LBH (the blue and green lines) and IBH+SBH (the brown line) in different scales. The green line is bounded by a critical point $\left(T_{c}, P_{c}\right)$ and point $\left(T_{f}, P_{f}\right)$ between SBH and LBH. Similarly, the blue line is bounded by this point $\left(T_{f}, P_{f}\right)$ and triple point $\left(T_{t}, P_{t}\right)$ between $\mathrm{SBH}$ and LBH. In addition, the brown line is bounded by this triple point $\left(T_{t}, P_{t}\right)$ and point $\left(T_{z}, P_{z}\right)$ between SBH and IBH. When black hole crosses the blue (brown) line from left to right or top to bottom, it undergoes a first (zeroth) order phase transition from SBH to LBH (IBH to SBH). So, one can see the RPT behavior of Born-Infeld-dilaton black holes for a narrow range of temperatures $T \in\left(T_{t}, T_{z}\right)$ and pressures $P \in\left(P_{t}, P_{z}\right)$. On the other hand, a zeroth order phase transition from SBH to LBH does occur whenever the black hole crosses the green line from left to right or top to bottom.

Table 1 shows some values for parameters $\alpha$ and $\beta$ which the black hole case study experiences the RPT investigated in this paper. We generated this table for fixed values of $q$ and $b$ due to the fact that these two parameters do not change the general behavior of the phase transition structure. From this table, one can find that the modification of zeroth order SBH-LBH phase transition below the critical point disappear whenever the dilaton parameter $\alpha$ is small enough. Thus, the dilaton field is responsible for this modification. In addition, we find that there is an $\alpha$, say $\alpha_{1}$, with $0.008<\alpha_{1}<0.02$ in which we have the common RPT of black holes for $\alpha<\alpha_{1}$

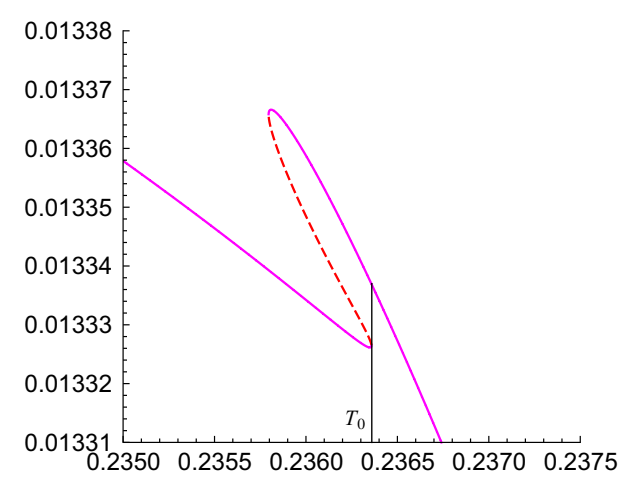

joins of dashed and solid lines, $C_{P}$ diverges. In addition, $G-T$ curves are shifted for clarity. The vertical line at $T_{1} \in\left(T_{t}, T_{z}\right)\left(T_{0} \in\left(T_{f}, T_{c}\right)\right)$ indicates a discontinuity in the Gibbs free energy which shows a zeroth order SBH-IBH (SBH-LBH) phase transition 


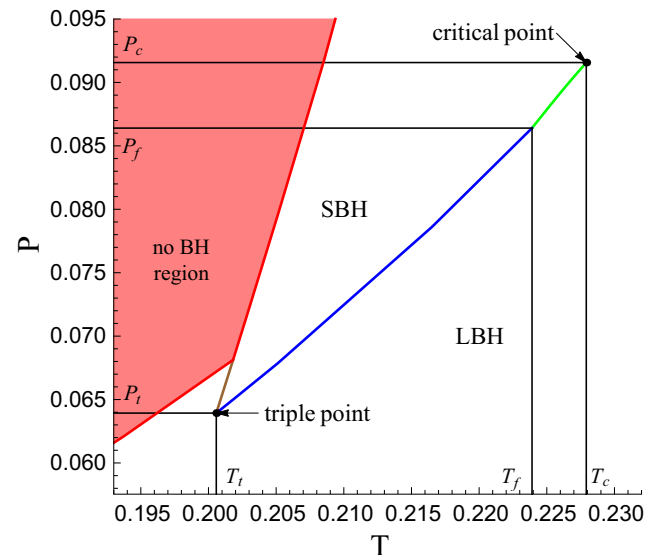

Fig. $3 P-T$ diagram. The shaded area illustrates the no black hole region and the right panel indicates a close-up of the RPT region. The blue and green curves indicate the coexistence lines of SBH and LBH, whereas the brown curve corresponds to the coexistence line of IBH

Table 1 The critical values for RPT for $q=0.2$ and $b=1$. The row specified with $*$ shows the common RPT of black holes in which there is no zeroth order SBH-LBH phase transition below the critical

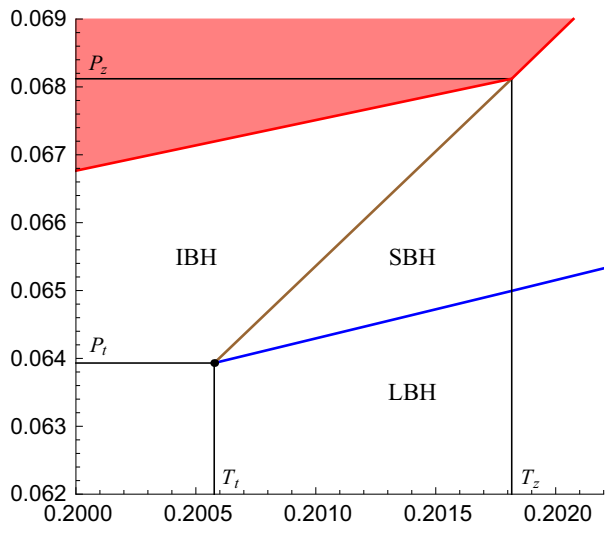

and SBH. On crossing the blue (brown) line, the system goes under a first (zeroth) order phase transition between SBH and LBH (SBH and IBH). In addition, on crossing the green line, the system undergoes a zeroth order SBH-LBH phase transition

point. The behavior of the system for the other rows is just like the case investigated throughout this paper

\begin{tabular}{llllllllll}
\hline$\beta$ & $\alpha$ & $r_{+c}$ & $T_{c}$ & $P_{c}$ & $\alpha$ & $\beta$ & $r_{+c}$ & $T_{c}$ & $P_{c}$ \\
\hline$* 2.000$ & $8.000 \times 10^{-3}$ & 0.4162 & 0.2294 & 0.09516 & $1.000 \times 10^{-1}$ & 1.850 & 0.4031 & 0.2310 & 0.09473 \\
2.000 & $2.000 \times 10^{-2}$ & 0.4165 & 0.2293 & 0.09504 & $1.000 \times 10^{-1}$ & 1.910 & 0.4132 & 0.2296 & 0.09327 \\
2.000 & $6.000 \times 10^{-2}$ & 0.4193 & 0.2289 & 0.09384 & $1.000 \times 10^{-1}$ & 1.970 & 0.4211 & 0.2284 & 0.09209 \\
2.000 & $1.000 \times 10^{-1}$ & 0.4245 & 0.2279 & 0.09157 & $1.000 \times 10^{-1}$ & 2.060 & 0.4304 & 0.2270 & 0.09065 \\
2.000 & $1.200 \times 10^{-1}$ & 0.4278 & 0.2273 & 0.09008 & $1.000 \times 10^{-1}$ & 2.120 & 0.4354 & 0.2262 & 0.08987 \\
2.000 & $1.400 \times 10^{-1}$ & 0.4314 & 0.2267 & 0.08840 & $1.000 \times 10^{-1}$ & 2.180 & 0.4397 & 0.2255 & 0.08918 \\
2.000 & $1.500 \times 10^{-1}$ & 0.4334 & 0.2264 & 0.08749 & $1.000 \times 10^{-1}$ & 2.240 & 0.4434 & 0.2249 & 0.08858 \\
\hline
\end{tabular}

and black holes undergo a zeroth order SBH-LBH phase transition below the critical point for $\alpha_{1}<\alpha<\alpha_{2}$ (we will obtain this $\alpha_{2}$ in coming section).

As a final remark of this section, we should note that based on our numerical analysis and plotted figures, and also the results of other related references, it seems that regardless of mass and electric charge, other hairs of a black hole (such as rotation, dilaton scalar field and etc) have considerable role for the existence of RPT.

\section{Zeroth order SBH-LBH phase transition}

In this section, we are going to show that our black hole case study can undergo a zeroth order SBH-LBH phase transition by adopting suitable parameters. This kind of phase transition is characterized by a finite jump in Gibbs energy similar to the one that has been shown in the last section. Such a phase transition has been obtained for dilaton black holes in [39] and it modifies the standard first order SBH-LBH phase transition.
In analogy manner with Ref. [41], here, we use the fixed values of $q=0.2, \alpha=0.6, \beta=2$, and $b=1$ to plot Figs. 4 and 5. The left panel of Fig. 4 shows isotherm curves of the system for different regions of temperature, and also, $G-T$ diagram of the system for different regions of pressure is illustrated in the right panel which these areas are equivalent to each other. For $T<T_{f}\left(P<P_{f}\right)$, the system undergoes the standard first order SBH-LBH phase transition which is similar to a van der Waals fluid. $T_{f}\left(P_{f}\right)$ is an upper bound for this behavior and the type of phase transition will change for $T_{f}<T<T_{c}\left(P_{f}<P<P_{c}\right)$. In this region, the order of phase transition will change into zeroth order. Finally, black holes undergo a second order SBH-LBH phase transition at the critical point $\left(T_{c}, P_{c}\right)$, and at temperatures and pressures above $T_{c}$ and $P_{c}$, SBH and LBH are physically indistinguishable. Therefore, the zeroth order SBH-LBH phase transition is located between the standard first order phase transition and critical point (see Fig. 5 for more details).

From Fig. 5, we find that the coexistence line of first order phase transition is nonlinear whereas the zeroth order segment looks linear. However, accurate numeric analysis 


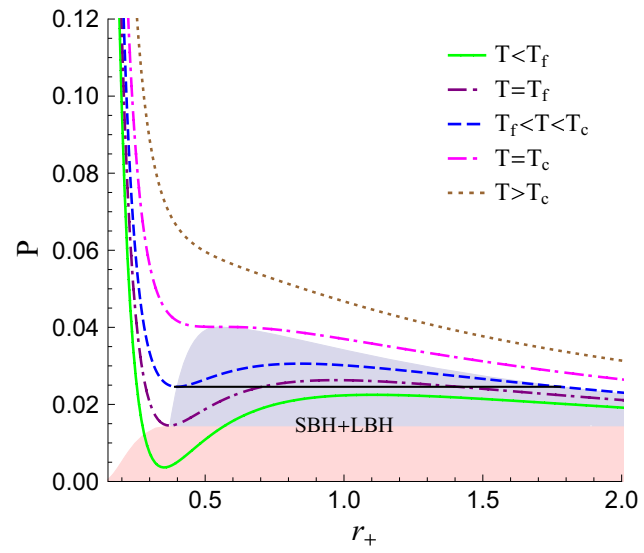

Fig. $4 P-r_{+}$and $G-T$ diagrams for different regions of temperature and pressure. In $P-r_{+}$diagram, the horizontal black line indicates a zeroth order SBH-LBH phase transition. Besides, the shaded area indicates $\mathrm{SBH}+\mathrm{LBH}$ coexistence region. In the right panel, the solid lines correspond to $C_{P}>0$ whereas the dashed red lines are related to

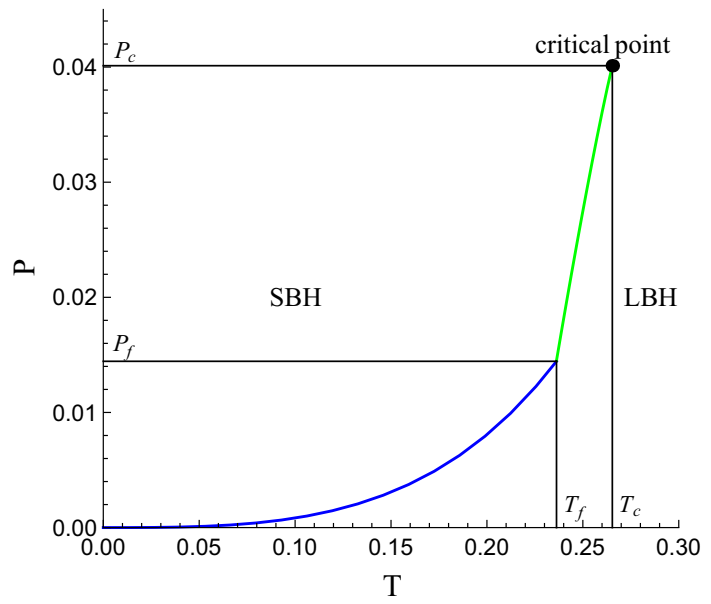

Fig. $5 P-T$ diagram. The blue and green curves indicate the coexistence line of SBH and LBH. On crossing the blue (green) line from left to right or top to bottom, the system goes under a first (zeroth) order phase transition from $\mathrm{SBH}$ to $\mathrm{LBH}$

Table 2 The critical values for zeroth order phase transition for $q=0.2$ and $b=1$. The row specified with $\dagger$ shows the RPT of black holes investigated in the previous section. For the row specified with $\ddagger$, the black hole does not experience the first order phase transition and there is just

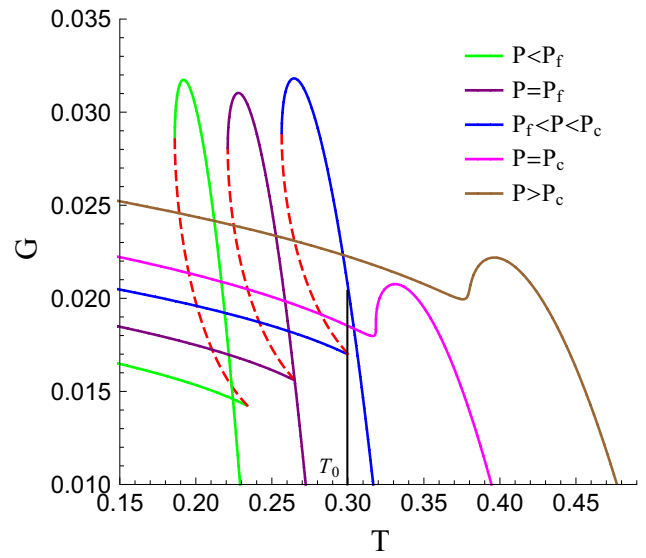

$C_{P}<0$. At the joins of dashed and solid lines, $C_{P}$ diverges. In addition, $G-T$ curves are shifted for clarity. The vertical line at $T_{0} \in\left(T_{f}, T_{c}\right)$, indicates a finite jump in the Gibbs energy which shows a zeroth order SBH-LBH phase transition

of the zeroth order phase transition shows that the related line segment in the $P-T$ diagram is not actually linear and our data show nonlinear behavior. This line segment is just like a very small part of a circle which looks as a line. On the other hand, the slope of the coexistence line represents the ratio of changes in entropy and volume, $\Delta S / \Delta r_{+}=$ $\left(S_{L}-S_{S}\right) /\left(r_{+L}-r_{+S}\right)$, where the subscripts $L$ and $S$ stand for $\mathrm{LBH}$ and $\mathrm{SBH}$, respectively. Therefore, we find that at the first order phase transition, the ratio $\Delta S / \Delta r_{+}$increases along the coexistence line when the temperature and pressure increase. But for the zeroth order case, the value of this ratio changes very slowly.

Table 2 shows some values for parameters $\alpha$ and $\beta$ which the black holes experience the zeroth order phase transition investigated in this section. From this table, we find that the range of $\alpha_{2}$, introduced in the previous section, with $0.15<$ $\alpha_{2}<0.2$. For $\alpha_{2}<\alpha<\alpha_{3}\left(0.9<\alpha_{3}<1\right)$ black hole experiences a zeroth order SBH-LBH phase transition for

zeroth order SBH-LBH phase transition. In addition, the value of $\alpha=1$ is undefined (see Eq. (17)) and the critical temperature is negative for $\alpha>1$. The behavior of the system for the other rows is just like the case investigated in this section

\begin{tabular}{|c|c|c|c|c|c|c|c|c|c|}
\hline$\beta$ & $\alpha$ & $r_{+c}$ & $T_{c}$ & $P_{c}$ & $\alpha$ & $\beta$ & $r_{+c}$ & $T_{c}$ & $P_{c}$ \\
\hline$\dagger 2.000$ & $1.500 \times 10^{-1}$ & 0.4334 & 0.2264 & 0.08749 & $6.000 \times 10^{-1}$ & 1.850 & 0.5444 & 0.2657 & 0.04028 \\
\hline 2.000 & $2.000 \times 10^{-1}$ & 0.4440 & 0.2248 & 0.08244 & $6.000 \times 10^{-1}$ & 1.910 & 0.5456 & 0.2655 & 0.04021 \\
\hline 2.000 & $4.000 \times 10^{-1}$ & 0.4928 & 0.2266 & 0.05933 & $6.000 \times 10^{-1}$ & 1.970 & 0.5467 & 0.2654 & 0.04015 \\
\hline 2.000 & $6.000 \times 10^{-1}$ & 0.5472 & 0.2654 & 0.04012 & $6.000 \times 10^{-1}$ & 2.060 & 0.5482 & 0.2653 & 0.04006 \\
\hline 2.000 & $9.000 \times 10^{-1}$ & 0.6471 & 0.8326 & 0.02279 & $6.000 \times 10^{-1}$ & 2.120 & 0.5490 & 0.2652 & 0.04002 \\
\hline$¥ 2.000$ & $9.900 \times 10^{-1}$ & 0.6827 & 7.988 & 0.01956 & $6.000 \times 10^{-1}$ & 2.180 & 0.5498 & 0.2651 & 0.03997 \\
\hline 2.000 & 1.100 & 0.7302 & -0.7725 & 0.01644 & $6.000 \times 10^{-1}$ & 2.240 & 0.5506 & 0.2650 & 0.03993 \\
\hline
\end{tabular}


a range of temperature and pressure. In addition, for $\alpha_{3}<$ $\alpha<1$ the standard first order phase transition segment will disappear and black holes just undergo a zeroth order phase transition. From Tables 1 and 2, one can see that the dilaton parameter strongly affects the phase transition behavior of the system.

\section{Conclusions}

In this paper, we have considered the cosmological constant as thermodynamical pressure and studied the thermodynamics of 4-dimensional Born-Infeld-dilaton black holes in the canonical ensemble of extended phase space. We have seen that in addition to the standard van der Waals like phase transition of these black holes [37,38], they can enjoy a novel RPT. It was shown that this behavior happens for a narrow range of temperatures and pressures. In this range of RPT, black holes undergo a zeroth order IBH-SBH phase transition and first order SBH-LBH phase transition. In addition, it was shown that there is a region below the critical point in which the first order phase transition modified to a zeroth order type. This behavior has never seen before in the RPT of black holes and we showed that it is due to the presence of the dilaton field.

Moreover, we have shown that by considering suitable values of free parameters, a portion of the standard first order SBH-LBH phase transition can modify into the zeroth order SBH-LBH phase transition. It is worthwhile to mention that numerical calculations show that this kind of phase transition is due to the presence of the dilaton field. It is interesting to investigate the microscopic origin of such RPT based on dilaton effect.

As the final remark, we should mention that three different behaviors have been found for the black hole case study presented in this paper until now. One is the standard van der Waals like phase transition presented in [37,38], and two of them are investigated throughout this paper. All these three behaviors depend on the choice of free parameters, and therefore, it may be possible to find some other different behaviors by adopting different values for the free parameters.

Acknowledgements The authors wish to thank the anonymous referee for the constructive comments that enhanced the quality of this paper. We wish to thank Shiraz University Research Council. This work has been supported financially by the Research Institute for Astronomy and Astrophysics of Maragha, Iran.

Open Access This article is distributed under the terms of the Creative Commons Attribution 4.0 International License (http://creativecomm ons.org/licenses/by/4.0/), which permits unrestricted use, distribution, and reproduction in any medium, provided you give appropriate credit to the original author(s) and the source, provide a link to the Creative Commons license, and indicate if changes were made.

Funded by SCOAP ${ }^{3}$.

\section{References}

1. P.C.W. Davies, Rep. Prog. Phys. 41, 1313 (1977)

2. P.C.W. Davies, Class. Quantum Grav. 6, 1909 (1989)

3. R.M. Wald, Liv. Rev. Rel. 4, 6 (2001)

4. S.W. Hawking, Commun. Math. Phys. 43, 199 (1975)

5. J.D. Bekenstein, Phys. Rev. D 7, 2333 (1973)

6. J.M. Bardeen, B. Carter, S.W. Hawking, Commun. Math. Phys. 31, 161 (1973)

7. S.W. Hawking, D.N. Page, Commun. Math. Phys. 87, 577 (1983)

8. A. Chamblin, R. Emparan, C.V. Johnson, R.C. Myers, Phys. Rev. D 60, 064018 (1999)

9. A. Chamblin, R. Emparan, C.V. Johnson, R.C. Myers, Phys. Rev. D 60, 104026 (1999)

10. J.Y. Shen, R.G. Cai, B. Wang, R.K. Su, Int. J. Mod. Phys. A 22, 11 (2007)

11. M.M. Caldarelli, G. Cognola, D. Klemm, Class. Quantum Grav. 17, 399 (2000)

12. D. Kastor, S. Ray, J. Traschen, Class. Quantum Grav. 26, 195011 (2009)

13. D. Kubiznak, R.B. Mann, JHEP 07, 033 (2012)

14. R. Banerjee, S.K. Modak, S. Samanta, JHEP 10, 125 (2012)

15. M.B.J. Poshteh, B. Mirza, Z. Sherkatghanad, Phys. Rev. D 88, 024005 (2013)

16. J.X. Mo, W.B. Liu, Eur. Phys. J. C 74, 2836 (2014)

17. D.C. Zou, Y.Q. Liu, B. Wang, Phys. Rev. D 90, 044063 (2014)

18. J. Xu, L.M. Cao, Y.P. Hu, Phys. Rev. D 91, 124033 (2015)

19. S.H. Hendi, S. Panahiyan, B. Eslam Panah, M. Faizal, M. Momennia, Phys. Rev. D 94, 024028 (2016)

20. B. Mandal, S. Samanta, B.R. Majhi, Phys. Rev. D 94, 064069 (2016)

21. S.H. Hendi, S. Panahiyan, M. Momennia, Int. J. Mod. Phys. D 25, 1650063 (2016)

22. S.H. Hendi, M. Momennia, Phys. Lett. B 777, 222 (2018)

23. S.H. Hendi, M. Momennia. arXiv: 1801.07906

24. C. Hudson, Z. Phys. Chem. Abt. A 47, 113 (1904)

25. T. Narayanan, A. Kumar, Phys. Rep. 249, 135 (1994)

26. S. Gunasekaran, R.B. Mann, D. Kubiznak, JHEP 11, 110 (2012)

27. N. Altamirano, D. Kubiznak, R.B. Mann, Phys. Rev. D 88, 101502(R) (2013)

28. M. Kord Zangeneh, A. Dehyadegari, A. Sheykhi, R.B. Mann. arXiv: 1709.04432

29. D. Kubiznak, F. Simovic, Class. Quantum Grav. 33, 245001 (2016)

30. R.A. Hennigar, R.B. Mann, Entropy 17, 8056 (2015)

31. D.C. Zou, R. Yue, M. Zhang, Eur. Phys. J. C 77, 256 (2017)

32. M. Zhang, D.C. Zou, R.H. Yue, Adv. High Energy Phys. 2017, 3819246 (2017)

33. R. Zhao, H.H. Zhao, M.S. Ma, L.C. Zhang, Eur. Phys. J. C 73, 2645 (2013)

34. J.X. Mo, G.Q. Li, X.B. Xu, Phys. Rev. D 93, 084041 (2016)

35. Z. Dayyani, A. Sheykhi, M.H. Dehghani, Phys. Rev. D 95, 084004 (2017)

36. H.F. Li, H.H. Zhao, L.C. Zhang, R. Zhao, Eur. Phys. J. C 77, 295 (2017)

37. S.H. Hendi, R. Moradi, Z. Armanfard, M.S. Talezadeh, Eur. Phys. J. C 76, 263 (2016)

38. M.H. Dehghani, A. Sheykhi, Z. Dayyani, Phys. Rev. D 93, 024022 (2016)

39. A. Dehyadegari, A. Sheykhi, A. Montakhab, Phys. Rev. D 96, 084012 (2017)

40. A. Sheykhi, Phys. Lett. B 662, 7 (2008)

41. Z. Dayyani, A. Sheykhi, M.H. Dehghani, S. Hajkhalili. arXiv:1709.06875 